\title{
On the road to large volumes in LM and SEM: New tools for Array Tomography
}

Irene Wacker ${ }^{1,2}$, Waldemar Spomer ${ }^{2,3}$, Andreas Hofmann ${ }^{2,3}$, Ulrich Gengenbach ${ }^{2,3}$, Marlene Thaler ${ }^{4}$, Len Ness ${ }^{5}$, Pat Brey ${ }^{5}$, and Rasmus R. Schröder ${ }^{2,6}$

${ }^{1}$ Cryo EM, Centre for Advanced Materials, Universität Heidelberg, Heidelberg, Germany

${ }^{2}$ HEiKA, Heidelberg Karlsruhe Research Partnership, Heidelberg, Karlsruhe, Germany

${ }^{3}$ Institute for Applied Computer Science, Karlsruhe Institute of Technology, Karlsruhe, Germany

${ }^{4}$ Carl Zeiss Microscopy GmbH, Oberkochen, Germany

${ }^{5}$ RMC Boeckeler, Tucson, Arizona, USA

${ }^{6}$ Cryo EM, CellNetworks, BioQuant, Universitätsklinikum Heidelberg, Heidelberg, Germany

To reconstruct large volumes at ultrastructural resolution a number of methods are available [1]. Serial blockface or focussed ion beam scanning electron microscopy (SBFSEM or FIBSEM) are inherently destructive: After many alternating cycles of blockface imaging and subsequent removal of a thin material layer to expose a new blockface the sample will be gone. Array tomography (AT) [2] on the other hand offers the possibility of imaging at different resolutions and with different techniques, such as (fluorescence) light microscopy (LM) and SEM. It therefore has the greatest potential for correlative imaging.

When aiming at large volumes, a high degree of automation during both, sample preparation and imaging procedure is desirable. One way to automate the generation of the thousands of serial sections necessary for brain mapping was introduced as ATUMtome [3] and is now commercially available for early adopters (RMC Boeckeler). Because the sections are collected on carbon-coated Kapton tape epifluorescence LM is possible in principle. However, super-resolution LM methods [4] do not work on such non-transparent tape.

To overcome this limitation we developed a novel device enabling reliable collection of long ribbons of serial sections (cf Spomer et al., this conference) onto a super-resolution LM-compatible solid substrate such as ITO-coated glass coverslips or onto silicon wafers for SEM imaging only.

Samples can be prepared either by classical aldehyde-based chemical fixation or by cryofixation and freeze substitution, both followed by epoxide embedding. For preservation of fluorescence signals or for post-embedding immuno-labeling, hydrophilic resins such as e.g. lowicryls are advisable. Blocks are trimmed and coated with a thin layer of glue on the leading and trailing edges to stabilize the ribbons. Substrates are hydrophilized by glow discharge and immersed into the boat of a Jumbo knife (Diatome) using the novel substrate holder. After cutting ribbons of ultrathin (down to 50nm) or semithin (up to $3 \mu \mathrm{m}$ ) sections, several ribbons are attached to the substrate (Fig. 1A) and gently lifted from the water. Fig. 1B shows the same four ribbons on a silicon wafer after lift-out and drying. Similarly, ITO-coated glass coverslips can be used as substrates (Fig. 1C).

Sections may be either stained with fluorescent dyes or antibodies for LM or with uranylacetate and lead citrate for SEM imaging. With sections up to $200 \mathrm{~nm}$ thickness high resolution SEM imaging is possible without further coating, using either secondary or back-scattered electrons.

To image large numbers of sections automation of the imaging process is necessary. The newly released product ZEISS Atlas 5 Array Tomography (Carl Zeiss Microscopy GmbH) allows repeated imaging of the sample at increasing resolution. Usually an overview of the whole substrate (Fig. 2A) is recorded first, at a pixel size of several 100nm. Then regions of interest (ROI), for example individual sections or parts thereof (Fig. 2B, C) are recorded with intermediate pixel sizes. Finally 
single cells or cell groups (Fig. 2D) can be selected and imaged automatically at pixel sizes down to 2-5 nm (Fig. 2C-D) and over large numbers of sections to reconstruct whole cells.

[1] I Wacker, RR Schröder, J Microscopy 252 (2013), p93-99.

[2] KD Micheva, DJ Smith, Neuron 55 (2007), p25-36. Erratum in: Neuron 55 (2007), p. 824.

[3] KJ Hayworth et al., Front Neural Circuits 8 (2014), article 68, p1-18.

[4] S Nanguneri, et al., PLoS One 7 (2012), e38098.

[5] The authors acknowledge C Bartels and L Veith for technical support, C Grabher for samples, BMBF for NanoCombine grant FKZ 13N11401 and MorphiQuant grant FKZ 13GW0044, and HEiKA for initial funding of the substrate holder development.
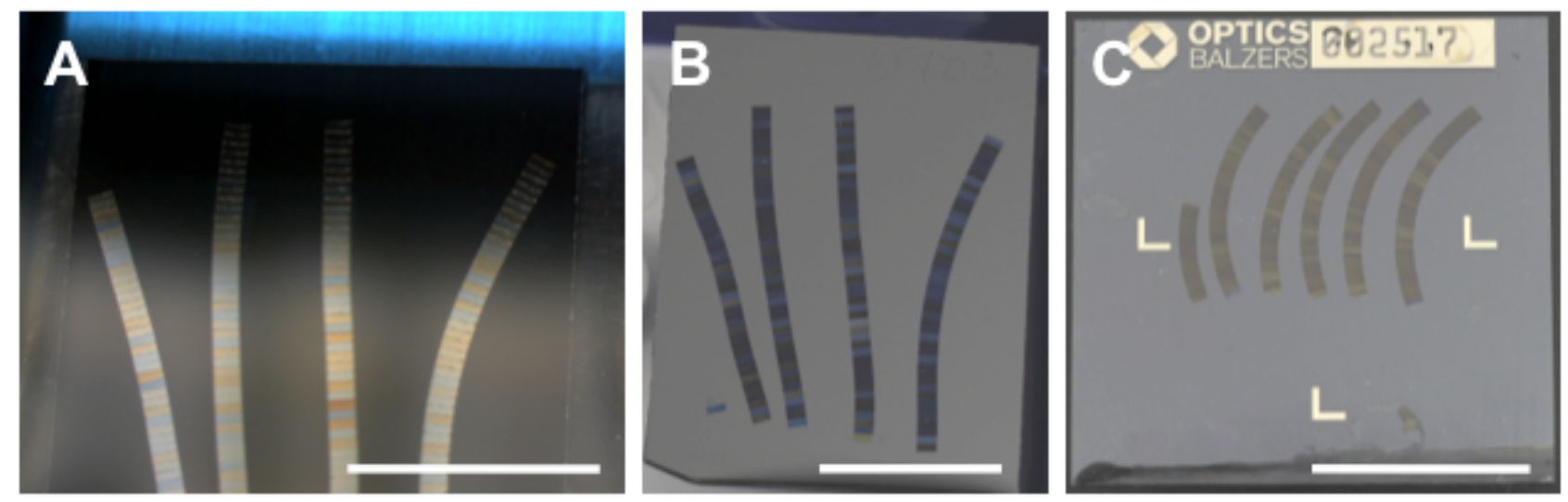

Fig. 1 Production of arrays of serial sections: A) Four ribbons floating in knife boat, attached to silicon wafer by their lower ends. B) The same ribbons (175 consecutive sections in total, about 40 sections per ribbon) dried onto wafer after lift-out. C) Six ribbons (about 200 sections in total) deposited between the fiducials (L-marks) on an ITO-coated glass coverslip. Scale bars $10 \mathrm{~mm}$
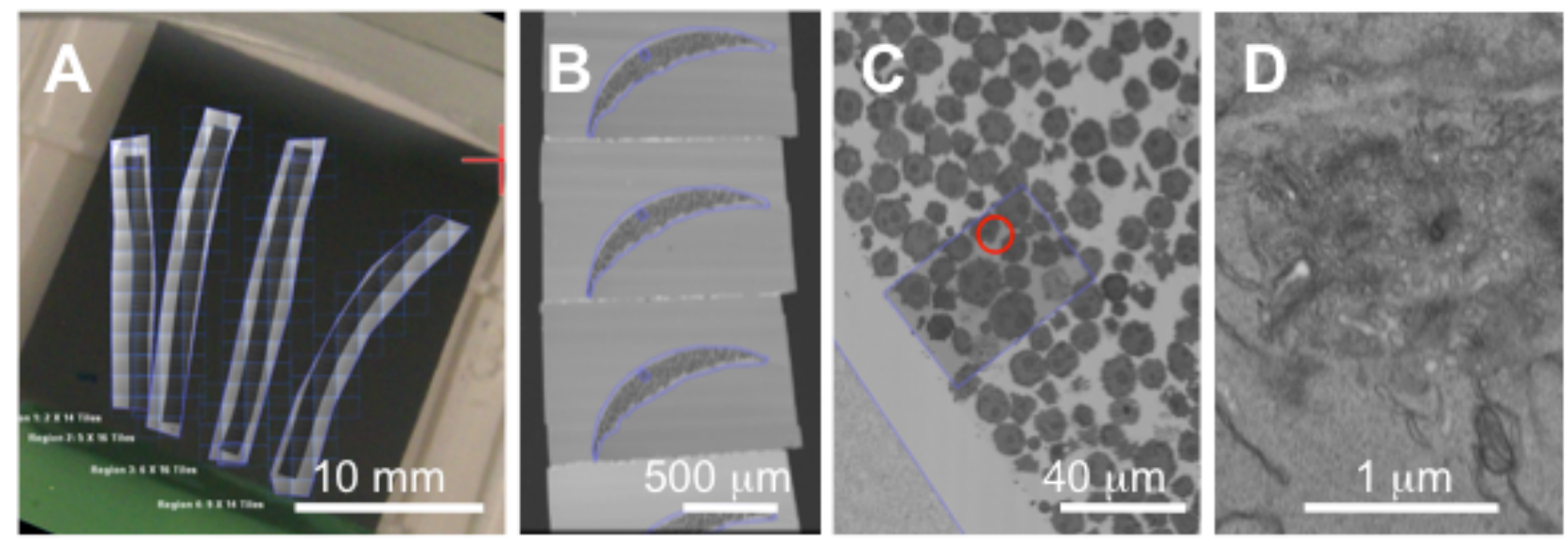

Fig. 2 Automatic imaging of arrays using ZEISS Atlas 5 Array Tomography solution: A) Overlay of ribbon images (SEM) on imported image of whole substrate (digital camera) used for initial navigation, B) individual sections, cell pellets (blue outline) imaged at 50nm pixel size, C) detail of B) with ROI (blue rectangle) imaged at $5 \mathrm{~nm}$ pixel size, D) Golgi region from cell marked by red circle in C) 